\title{
Le Comité international de bioéthique (CIB) de I'UNESCO et la Déclaration universelle sur la bioéthique et les droits de l'homme
}

\section{Jean Martin}

Membre du Comité international de bioéthique et de la Commission nationale suisse d'éthique pour la médecine humaine
Correspondance:

Dr Jean Martin

Ancien médecin cantonal

La Ruelle 6

CH-1026 Echandens

jean.martin@urbanet.ch

\section{Le CIB}

L'UNESCO, organisation des Nations Unies pour l'éducation, la science et la culture dont le siège est à Paris, est amenée par son mandat à se préoccuper d'éthique (www.unesco.org/bioethics). Son Directeur général actuel, Koïchiro Matsuura, en a fait une de ses priorités. C'est dans ce cadre que, en 1993, a été créé le Comité international de bioéthique (CIB), formé de 36 personnalités du monde entier, nommées par le Directeur général sur présentation des gouvernements mais siégeant ad personam. Les membres, désignés pour quatre ans (ils ne peuvent l'être pour plus de deux mandats consécutifs), appartiennent à différents domaines: sciences de la vie, sciences sociales et humaines (notamment juridiques), droits de l'homme, philosophie, éducation et communication. En plus de cette pluridisciplinarité, la composition du CIB doit répondre à des critères de diversité culturelle et de répartition géographique.

Les fonctions principales du CIB sont de favoriser la réflexion sur les enjeux éthiques et juridiques des recherches dans les sciences de la vie et de leur applications, encourager des actions de sensibilisation à la bioéthique de l'opinion publique, des milieux spécialisés et des décideurs, coopérer avec les organisations intergouvernementales (OIG) et non gouvernementales (ONG) concernées, ainsi qu'avec les comités nationaux et régionaux de bioéthique, et contribuer à la mise en œuvre des recommandations émises par l'UNESCO, en particulier:

- la Déclaration universelle sur le génome humain et les droits de l'homme (1997);

- la Déclaration internationale sur les données génétiques humaines (2003).

Par l'élaboration d'instruments internationaux tels que ces Déclarations, le CIB contribue à une action normative promouvant un cadre éthique commun que les Etats peuvent utiliser pour définir leurs politiques en la matière. Au reste, dès 1994, le Comité a publié plus d'une quinzaine de rapports, notamment sur les questions liées à la génétique (tests et données y compris confiden- tialité et stockage, conseil, recherche, thérapie), la propriété intellectuelle, la brevetabilité et la coopération internationale en génomique, le diagnostic préimplantatoire, les cellules souches, les neurosciences, l'alimentation et les biotechnologies végétales.

L'expérience acquise durant les premières années d'activité du CIB a montré le besoin de créer une autre instance, le Comité intergouvernemental de bioéthique (CIGB), aussi formé de 36 membres mais qui sont eux des représentants des Etats-membres de l'UNESCO, qui apportent donc les appréciations politiques de leurs gouvernements. Les deux Comités peuvent se réunir en session conjointe, afin de favoriser le dialogue entre eux sur des sujets d'intérêt commun et s'efforcer de résoudre d'éventuelles divergences.

Depuis le début 2006, la présidente du CIB est Madame le Professeur Nouzha Guessous Idrissi, chef du Service de parasitologie-mycologie et membre du Comité d'éthique, Faculté de médecine et de pharmacie de Casablanca (Maroc); elle a remplacé Madame Michèle Jean, ancienne sous-ministre de la Santé du Canada, membre du Comité permanent d'éthique des Instituts canadiens de recherche en santé. En rapport avec la Suisse, on peut noter que le Dr Roberto Andorno, ancien professeur de droit civil à l'Université de Buenos Aires et actuellement chercheur au Centre d'éthique de l'Université de Zurich, en a fait partie jusqu'en 2005. L'auteur du présent article a été désigné membre du CIB en mars 2006.

\section{Commissions nationales pour I'UNESCO}

Il est intéressant de noter que l'UNESCO est la seule agence des Nations Unies à avoir un système de Commissions nationales dans ses quelque 190 Etats-membres. Ces commissions ont pour vocation d'être un lien entre la société civile et l'organisation, assistant dans la mise en œuvre d'initiatives telles que programmes de formation, études, actions de sensibilisation et de communication. Les commissions nationales peuvent développer des partenariats avec le secteur privé. La Commission nationale suisse pour l'UNESCO, qui est présidée par l'avocate tessi- 
noise Francesca Gemnetti, dispose d'un secrétariat au Département fédéral des affaires étrangères. De plus la Suisse œuvre sur place, au siège de l'organisation à Paris, par un délégué permanent, actuellement l'ambassadeur Ernst Iten.

\section{La Déclaration universelle sur la bioéthi- que et les droits de I'homme de $\mathbf{2 0 0 5}$}

Après des travaux préparatoires qui ont duré deux ans et ont donné lieu à débats et consultations, notamment par le canal des Commissions nationales qui viennent d'être évoquées, la Conférence générale de l'UNESCO a adopté cette

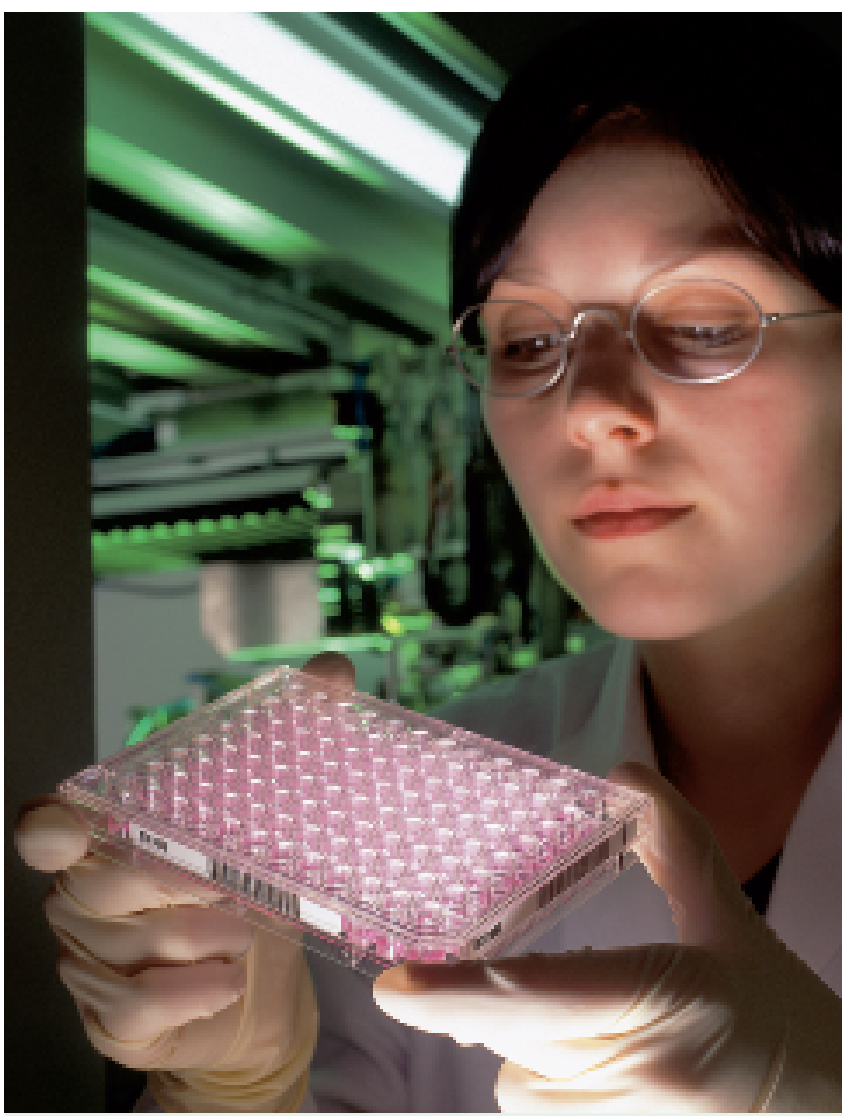

L'évolution des sciences biomédicales engendre des questions éthiques.

Déclaration en octobre 2005. Son importance et son utilité sont de constituer un document accepté par l'ensemble des pays quant aux principes qui doivent régir les droits des personnes, particulièrement des patients, dans le domaine des soins de santé et de la recherche biomédicale. Elle s'appuie sur des instruments internationaux existants, notamment la Convention sur les droits de l'homme et la biomédecine du Conseil de l'Europe de 1997, avec ses Protocoles additionnels, et des codes de conduite tels que la Déclaration d'Helsinki de l'Association médicale mondiale (AMM), adoptée en 1964 et amendée à plusieurs reprises - la dernière fois en 2000 , ainsi que les Principes d'éthique de la recherche biomédicale du Conseil des organisations internationales des sciences médicales (CIOMS).

Dans son préambule, dans des termes qui sont ceux du langage diplomatique propre à ce type d'instrument international mais qui méritent d'être relevés, la Déclaration souligne qu'il est nécessaire et qu'il est temps que la communauté internationale énonce des principes universels sur la base desquels l'humanité pourra répondre aux dilemmes et controverses de plus en plus nombreux que la science et la technologie suscitent pour l'humanité et l'environnement. Elle rappelle que les êtres humains font partie intégrante de la biosphère et ont un rôle important à jouer en se protégeant les uns les autres et en protégeant les autres formes de vie, et que l'identité de la personne a des dimensions biologiques, psychologiques, sociales, culturelles et spirituelles. Et elle affirme que la sensibilité morale et la réflexion éthique devraient faire partie intégrante du processus de développement scientifique et technologique; la bioéthique a un rôle capital à jouer dans les choix à faire face aux problèmes qu'entraîne ce développement.

Suivent 28 articles de la Déclaration stricto sensu, dont il est approprié ici de citer plusieurs passages:

Article premier - Portée

1. La présente Déclaration traite des questions d'éthique posées par la médecine, les sciences de la vie et les technologies qui leur sont associées, appliquées aux êtres humains, en tenant compte de leurs dimensions sociale, juridique et environnementale (...).

Article 2-Objectifs

La présente Déclaration a pour objectifs:

(a) d'offrir un cadre universel de principes et de procédures pour guider les États dans la formulation de leur législation, de leurs politiques ou d'autres instruments en matière de bioéthique;

(b) de guider les actions des individus, des groupes, des communautés, des institutions et des sociétés, publiques et privées;

(c) de contribuer au respect de la dignité humaine et de protéger les droits de l'homme, en assurant le respect de la vie des êtres humains, et les libertés fondamentales, d'une manière compatible avec le droit international des droits de l'homme;

(d) de reconnaître l'importance de la liberté de la recherche scientifique et des bienfaits découlant des progrès des sciences et des technologies, tout en insistant sur la nécessité pour cette recherche et ces progrès de s'inscrire dans le cadre des principes éthiques énoncés dans la présente Déclaration et de respecter la dignité humaine, les droits de l'homme et les libertés fondamentales;

(e) d'encourager un dialogue pluridisciplinaire et pluraliste sur les questions de bioéthique entre toutes les parties intéressées et au sein de la société dans son ensemble; 
(f) de promouvoir un accès équitable aux progrès de la médecine, des sciences et des technologies, ainsi que la plus large circulation possible et un partage rapide des connaissances concernant ces progrès et le partage des bienfaits qui en découlent, en accordant une attention particulière aux besoins des pays en développement;

(g) de sauvegarder et défendre les intérêts des générations présentes et futures;

(h) de souligner l'importance de la biodiversité et de sa préservation en tant que préoccupation commune à l'humanité.

Article 3 - Dignité humaine et droits de l'homme

1. La dignité humaine, les droits de l'homme et les libertés fondamentales doivent être pleinement respectés.

2. Les intérêts et le bien-être de l'individu devraient l'emporter sur le seul intérêt de la science ou de la société.

Article 5 - Autonomie et responsabilité individuelle L'autonomie des personnes pour ce qui est de prendre des décisions, tout en en assumant la responsabilité et en respectant l'autonomie d'autrui, doit être respectée. Pour les personnes incapables d'exercer leur autonomie, des mesures particulières doivent être prises pour protéger leurs droits et intérêts.

Article 6 - Consentement

1. Toute intervention médicale de caractère préventif, diagnostique ou thérapeutique ne doit être mise en œuvre qu'avec le consentement préalable, libre et éclairé de la personne concernée, fondé sur des informations suffisantes. Le cas échéant, le consentement devrait être exprès et la personne concernée peut le retirer à tout moment et pour toute raison sans qu'il en résulte pour elle aucun désavantage ni préjudice.

2. Des recherches scientifiques ne devraient être menées qu'avec le consentement préalable, libre, exprès et éclairé de la personne concernée. L'information devrait être suffisante, fournie sous une forme compréhensible et indiquer les modalités de retrait du consentement. La personne concernée peut retirer son consentement à tout moment et pour toute raison sans qu'il en résulte pour elle aucun désavantage ni préjudice (...).

Article 7 - Personnes incapables d'exprimer

leur consentement

En conformité avec le droit interne, une protection spéciale doit être accordée aux personnes qui sont incapables d'exprimer leur consentement:

(a) l'autorisation d'une recherche ou d'une pratique médicale devrait être obtenue conformément à l'intérêt supérieur de la personne concernée et au droit interne. Cependant, la personne concernée devrait être associée dans toute la mesure du possible au processus de décision conduisant au consentement ainsi qu'à celui conduisant à son retrait;

(b) une recherche ne devrait être menée qu'au bénéfice direct de la santé de la personne concernée, sous réserve des autorisations et des mesures de protection prescrites par la loi et si il n'y a pas d'autre option de recherche d'efficacité comparable faisant appel à des participants capables d'exprimer leur consentement (...).

Article 8 - Respect de la vulnérabilité humaine et de l'intégrité personnelle

Dans l'application et l'avancement des connaissances scientifiques, de la pratique médicale et des technologies qui leur sont associées, la vulnérabilité humaine devrait être prise en compte. Les individus et les groupes particulièrement vulnérables devraient être protégés et l'intégrité personnelle des individus concernés devrait être respectée.

Article 9 - Vie privée et confidentialité

La vie privée des personnes concernées et la confidentialité des informations les touchant personnellement devraient être respectées. Dans toute la mesure du possible, ces informations ne devraient pas être utilisées ou diffusées à des fins autres que celles pour lesquelles elles ont été collectées ou pour lesquelles un consentement a été donné (...)

Article 12 - Respect de la diversité culturelle et du pluralisme

Il devrait être tenu dûment compte de l'importance de la diversité culturelle et du pluralisme. Toutefois, ces considérations ne doivent pas être invoquées pour porter atteinte à la dignité humaine, aux droits de l'homme et aux libertés fondamentales ou aux principes énoncés dans la présente Déclaration, ni pour en limiter la portée.

Article 14 - Responsabilité sociale et santé

1. La promotion de la santé et du développement social au bénéfice de leurs peuples est un objectif fondamental des gouvernements que partagent tous les secteurs de la société (...)

Article 15 - Partage des bienfaits

1. Les bienfaits résultant de toute recherche scientifique et de ses applications devraient être partagés avec la société dans son ensemble ainsi qu'au sein de la communauté internationale, en particulier avec les pays en développement (...)

2. Les bienfaits ne devraient pas constituer des incitations inappropriées à participer à la recherche.

Article 16 - Protection des générations futures

L'incidence des sciences de la vie sur les générations futures, y compris sur leur constitution génétique, devrait être dûment prise en considération.

Article 17 - Protection de l'environnement,

de la biosphère et de la biodiversité

Il convient de prendre dûment en considération l'interaction entre les êtres humains et les autres formes de vie, de même que l'importance d'un accès approprié aux ressources biologiques et génétiques et d'une utilisation appropriée de ces ressources, le respect des savoirs traditionnels, ainsi que le rôle des êtres humains dans la protection de l'environnement, de la biosphère et de la biodiversité.

Article 18 - Prise de décisions et traitement des questions de bioéthique

1. Le professionnalisme, l'honnêteté, l'intégrité et la transparence dans la prise de décisions devraient être encouragés, en particulier la déclaration de tout conflit d'intérêts et un partage approprié des connaissances (...)

2. Un dialogue devrait être engagé de manière régulière entre les personnes et les professionnels concernés ainsi que la société dans son ensemble (...)

Article 19 - Comités d'éthique

Des comités d'éthique indépendants, pluridisciplinaires et pluralistes devraient être mis en place, encouragés et soutenus, au niveau approprié (...).

Article 23 - Education, formation et information en matière de bioéthique

Afin de promouvoir les principes énoncés dans la présente Déclaration et d'assurer une meilleure com- 
préhension des enjeux éthiques liés aux progrès des sciences et des technologies, en particulier chez les jeunes, les États devraient s'efforcer de favoriser l'éducation et la formation en matière de bioéthique à tous les niveaux, et d'encourager les programmes d'information et de diffusion des connaissances concernant la bioéthique (...).

\section{En Suisse}

D'abord, il convient de rappeler la désignation, par le Conseil fédéral en juillet 2001, de la Commission nationale d'éthique pour la médecine humaine (CNE-NEK), sur la base de la loi fédérale sur la procréation médicalement assistée. Depuis lors, cette commission s'est penchée sur de nombreux thèmes actuels d'importance, parfois controversés - à plusieurs reprises en lien avec des enjeux juridiques et législatifs - et a émis 13 prises de position jusqu'à fin 2006 (voir site www.nek-cne.ch). On rappellera aussi, en matière bioéthique, les contributions importantes de l'Académie Suisse des Sciences Médicales (ASSM), par les recommandations et directives qu'elle émet.

En rapport avec la Déclaration universelle présentée ici, il faut relever la mise en consultation en 2006, par le Conseil fédéral, d'un projet de loi relative à la recherche sur l'être humain, à propos duquel la CNE s'est prononcée favorablement tout en émettant quelques réserves [1]. En général, la Suisse suit de près les réflexions au niveau international et les textes élaborés chez nous sont dans la ligne des options et déclarations internationales.

\section{Expérience personnelle}

Depuis sa création, j'ai le privilège, avec beaucoup d'intérêt, de participer aux travaux de la Commission nationale d'éthique. Désigné au début 2006 au sein du CIB de l'UNESCO, j'ai pour l'instant participé à deux réunions. En juin 2006, au Siège de l'UNESCO à Paris, à un Groupe de travail ayant pour mission d'élaborer un document explicatif et illustratif sur la question du consentement (art. 6 et 7 de la Déclaration universelle). Et, en novembre 2006, à la $13^{\mathrm{e}}$ Session du CIB, où ont été discutés un avant-projet du texte sur le consentement ainsi que le thème de la responsabilité sociale en matière de santé (art. 14 de la Déclaration). A noter que les sessions plénières $\mathrm{du}$ CIB sont publiques et qu'elles permettent ainsi des échanges fructueux avec les milieux intéressés (sociétés scientifiques et professionnelles, ONG, etc.).

PS: les actes des sessions du CIB et d'autres documents concernant son activité peuvent être obtenus à l'adresse suivante: UNESCO, Division de l'éthique des sciences et des technologies, Section de bioéthique, 7, Place de Fontenoy, F-75352 Paris 07 SP, ou par e-mail: s.colombo@ unesco.org.

\section{Référence}

1 CNE. Recherche sur l'être humain: bon projet de loi, mais quelques bémols. Bull Méd Suisses. 2006;87(27/28):1268. 